\title{
Discrepancies between observational and theoretical parameters for three O-type binaries
}

\author{
Laura R. Penny ${ }^{1}$, Douglas R. Gies ${ }^{2}$, and W.G. Bagnuolo $\mathrm{Jr}^{2}$ \\ ${ }^{1}$ Department of Physics and Astronomy, College of Charleston, \\ Charleston, SC 29424, USA \\ ${ }^{2}$ CHARA and Department of Physics and Astronomy, \\ Georgia State University, Atlanta, GA 30303, USA
}

\begin{abstract}
Here we present fundamental parameters (masses, spectral classifications, rotational velocities) obtained from a combination of spectroscopic and photometric orbital solutions for the O-type binary systems HD 152248, HD 149404, and HD 135240. We also present estimated masses derived from theoretical single star evolutionary tracks for these same stars. In all cases the evolutionary masses are larger than those derived from observations.
\end{abstract}

\section{Introduction}

Observational masses from a combination of photometric and spectroscopic orbits of close, massive binaries can be used as constraints for theoretically determined masses. The three O-type binary systems presented here all have observed masses below those predicted by evolutionary models for single stars. All three systems (HD 152248, HD 135240, and HD 149404) are well known, double-lined spectroscopic binaries with some photometric variability.

\section{Spectroscopic orbits}

We obtained the data from IUE, high dispersion, short wavelength camera. Cross-correlation analysis was performed using a narrow-lined template star (HD 152248 and HD 34078 (O9.5 V); HD 135240 and HD 149438 (BO V); and HD 149404 and HD 46202(O9 V)). This analysis results in radial velocities, projected rotational velocities (Penny 1996), and the ultraviolet flux ratio (Penny, Gies \& Bagnuolo 1997). Orbital elements are obtained from the SBCM-code of Morbey \& Brosterhus (1974). We present the orbital parameters in Table 1. In the case of HD 135240, we adopted the orbital parameters of Stickland et al. (1993). We also adopted the period found by Stickland \& Koch (1996) for HD 149404.

\section{Photometric orbits}

Light-curve models are calculated with the GENSYN-code of Mochnacki et al. (1986). In order to calculate the model fits, we require estimates of the orbital elements (spectroscopic orbit), the effective temperatures of the stars (spectral types from the method described in Penny, Gies \& Bagnuolo 1996 and the effective temperature - spectral type calibration of Howarth \& Prinja 1989), and the 
Table 1. Parameters for HD 152248, HD 135240 and HD 149404.

\begin{tabular}{|c|c|c|c|}
\hline parameter & HD 152248 & HD 135240 & HD 149404 \\
\hline inclination $\left({ }^{\circ}\right)$ & $72(3)$ & $77(2)$ & $30(5)$ \\
\hline period (days) & $5.816083(18)$ & 3.902456 (fixed) & 9.81452 (fixed) \\
\hline $\mathrm{T}(\mathrm{HJD} 2400000+$ ) & $44122.7(1)$ & 48884.285 (fixed) & $44449.38(7)$ \\
\hline$e$ & $0.13(2)$ & 0.061 (fixed) & 0.0 (fixed) \\
\hline$\omega\left({ }^{\circ}\right)$ & $57.3(7.5)$ & 274.4(fixed) & 0.0 (fixed) \\
\hline$K_{1}\left(\mathrm{~km} \mathrm{~s}^{-1}\right)$ & $215.5(2.8)$ & 148.4(fixed) & $86.6(2.1)$ \\
\hline$K_{2}\left(\mathrm{~km} \mathrm{~s}^{-1}\right)$ & $202.2(3.9)$ & 257.4 (fixed) & $74.0(3.0)$ \\
\hline$v_{0}\left(\mathrm{~km} \mathrm{~s}^{-1}\right)$ & $-24.7(2.5)$ & $12.5(2.0)$ & $-56.8(2.0)$ \\
\hline$a\left(10^{6} \mathrm{~km}\right)$ & $33.8(1.4)$ & $22.3(9)$ & $43.4(7.2)$ \\
\hline \multicolumn{4}{|l|}{ UV spectral class } \\
\hline (primary) & O7 I & O7 V & O8.5 I \\
\hline (secondary) & O7 I & O9 V & O6.5 III \\
\hline$T_{1}(\mathrm{kK})$ & $35(1.5)$ & $37.5(2.0)$ & $32(1.0)$ \\
\hline$T_{2}(\mathrm{kK})$ & $37(1.5)$ & $35(2.0)$ & $39(3.0)$ \\
\hline $\log L_{1} / \mathrm{L}_{\odot}$ & $5.47(8)$ & $5.16(10)$ & $5.73(9)$ \\
\hline $\log L_{1} / L_{\odot}$ & $5.53(8)$ & $4.37(26)$ & $5.74(15)$ \\
\hline$v_{1 \text { rot }}\left(\mathrm{km} \mathrm{s}^{-1}\right)$ & 172 & 159 & 172 \\
\hline$v_{2 r o t}\left(\mathrm{~km} \mathrm{~s}^{-1}\right)$ & 182 & 82 & 109 \\
\hline \multicolumn{4}{|l|}{ observational masses } \\
\hline$M_{1} / \mathrm{M}_{\odot}$ & $24.2(2.0)$ & $18.5(7)$ & $16(8)$ \\
\hline $\begin{array}{l}M_{2} / \mathrm{M}_{\odot} \\
\text { evolutionary masses }\end{array}$ & $25.8(2.0)$ & $10.7(4)$ & $18(9)$ \\
\hline$M_{1} / \mathrm{M}_{\odot}$ & $35.1(3.3)$ & $31.5(4.5)$ & $43.7(5.3)$ \\
\hline$M_{2} / \mathrm{M}_{\odot}$ & $38.0(4.5)$ & $16.9(4.0)$ & $49.3(12.0)$ \\
\hline
\end{tabular}

flux ratio between the stars (cross-correlation analysis). Before spectral classification can begin, we must perform a tomographic separation of the composite spectra to obtain the individual component spectra of the stars (see Bagnuolo et al. 1994). Each model light-curve is then a function of the orbital inclination and the primary polar radius. The results of the best light-curve model fits are presented in Table 1, along with the results of combining the photometric and spectroscopic orbits, and theoretical masses calculated from the single stellar evolutionary tracks of Schaller et al. (1992). None of the stars is currently filling its Roche-volume.

\section{References}

Bagnuolo, W.G., Gies, D.R., Hahula, M.E., Wiemker, R., Wiggs, M.S. 1994, ApJ 423, 446

Mochnacki, S.W., Fernie, J.D., Lyons, R., Schmidt, F.H., Gray, R.O. 1986, AJ 91, 1221 Morbey, C., Brosterhus, E.B. 1974, PASP 86, 455

Penny, L.R. 1996, ApJ 463, 737

Penny, L.R., Gies, D.R., Bagnuolo, W.G. 1996, ApJ 460, 906

Penny, L.R., Gies, D.R., Bagnuolo, W.G. 1997, ApJ 483, 439

Schaller, G., Schaerer, D., Meynet, G., Maeder, A. 1992, A\&AS 96, 269

Stickland, D.J., Koch, R.H., Pachoulakis, I., Pfeiffer, R.J. 1993, Observatory 113, 139

Stickland, D.J., Koch, R.H. 1996, Observatory 116, 145 